FORMATION Formation emploi

Revue française de sciences sociales

147 | Juillet-Septembre

Italie : réformes politiques et permanences sociétales des relations formation-emploi

\title{
Nous ne sommes plus des travailleuses, nous sommes des femmes de cinquante ans!
}

We are no longer workers, we are fifty-year-old women!

Wir sind keine Arbeiterinnen mehr, wir sind fünfzig Jahre alte Frauen!

¡Ya no somos trabajadoras, somos mujeres de cincuenta años!

\section{Tania Toffanin}

\section{(2) OpenEdition}

Journals

Édition électronique

URL : http://journals.openedition.org/formationemploi/7651

DOI : 10.4000/formationemploi.7651

ISSN : 2107-0946

Éditeur

La Documentation française

Édition imprimée

Date de publication : 31 octobre 2019

Pagination : 113-132

ISSN : 0759-6340

Référence électronique

Tania Toffanin, « Nous ne sommes plus des travailleuses, nous sommes des femmes de cinquante ans ! », Formation emploi [En ligne], 147 | Juillet-Septembre, mis en ligne le 03 janvier 2021, consulté le 02 mars 2021. URL : http://journals.openedition.org/formationemploi/7651 ; DOI : https://doi.org/ 10.4000/formationemploi.7651 


\title{
Nous ne sommes plus des travailleuses, nous sommes des femmes de cinquante ans !
}

\author{
TANIA TOFFANIN \\ Sociologue de l'économie et du travail, Université de Padoue, Département de philosophie, \\ pédagogie, sociologie et psychologie appliquée
}

Résumé

\begin{abstract}
Nous ne sommes plus des travailleuses, nous sommes des femmes de cinquante ans !
\end{abstract}

En Italie, la demande de personnel dans les secteurs à forte intensité de main-d'œuvre, tels que le textile, l'habillement et la chaussure, a diminué en raison des délocalisations de ces activités. Dans le même temps, les gouvernements italiens ont progressivement reporté l'âge de la retraite, mais sans modification structurelle des politiques actives du marché du travail. Aussi, pour les travailleuses plus âgées ayant un faible niveau d'éducation, le risque est de connaître une instabilité du revenu jusqu'à la retraite et une pension de retraite réduite. En mobilisant une cinquantaine d'entretiens auprès de travailleuses âgées licenciées, de partenaires sociaux, de représentants politiques et de fonctionnaires, nous analysons la condition de ces travailleuses et les facteurs qui freinent leur intégration dans le marché du travail.

Mots clés : emploi des femmes, chômage des femmes, travailleur âgé, BNQ - bas niveau de qualification -, recherche d'emploi, politique de l'emploi, mesure pour l'emploi, marche local du travail, disparité régionale, accès à la formation professionnelle, relation travail-famille, Italie

Abstract

We are no longer workers, we are fifty-year-old women!

In Italy, the labour demand in labour intensive sectors, such as textile, clothing and footwear, has gradually declined due to the effects of the relocation of the low-added value activities in low-wage countries. At the same time, Italian governments have progressively postponed the retirement age but without the implementation of structural interventions in active labour market policies. Consequently, for older women workers with a low level of education, the risk is to remain in a state of income instability until retirement while the amount of their pension will be necessarily reduced. Based on the analysis of fifty interviews carried out with dismissed older women workers, social par- 
tners and public officers, we analyse the conditions of these workers and the factors that prevent their integration into the labour market.

Keywords: women's employment, female unemployment, elderly worker, low level qualifications, ob search ; employment policy, employment scheme, local labour market, regional disparity, access to cvt, work-family relationship, Italy

Journal of Economic Literature: J 68 ; J 64

Traduction : auteure.

L'analyse du statut des travailleuses est généralement réalisée sur la population féminine âgée de 25 à 49 ans. À l'inverse, celle relative au statut des travailleuses de plus de 50 ans est encore rare, en particulier dans les pays méditerranéens. Les raisons de cette lacune sont principalement dues à la longue invisibilisation des femmes âgées sur le marché du travail. Dans les pays méditerranéens, l'organisation de la protection sociale, la structure économique et la culture patriarcale ont engendré un système spécifique en termes de rôle travail-famille (Pleck, 1977). Ce système est fondé sur un travail domestique et du care (soins prodigués à des proches) important et principalement réservé aux femmes, des taux élevés d'inactivité féminine, des discontinuités des carrières des femmes en raison de la maternité ou des soins prodigués à des membres qui sont à la charge de la famille, et des emplois en inadéquation avec les besoins en termes de conciliation vie professionnelle/ vie privée.

En Italie, ces facteurs ont fortement limité les taux de participation des femmes au travail rémunéré, en particulier des femmes âgées. Cependant, progressivement, avec l'évolution de la société et les réformes des retraites, le nombre de femmes âgées de 50 ans et plus sur le marché du travail a augmenté. Néanmoins, comme en attestent les données Eurostat, les femmes les plus instruites sont les plus concernées par cette croissance : le taux d'emploi des femmes âgées de 55 à 64 ans et diplômées du supérieur est passé de 54,8 \% en 2005 à 78,3\% en 2018 (dans l'UE, ce taux est passé de 57,4\% à 69,3\%). En revanche, le taux d'emploi des femmes âgées de 55 à 64 ans et ayant un niveau d'études primaires a augmenté, passant de $14 \%$ en 2005 à $27 \%$ en 2018 (dans l'UE, ce taux est passé de $26,8 \%$ à 36,5 \%), tandis que parmi ces dernières, en 2018, le taux de chômage a atteint $9,3 \%$ (contre $1,8 \%$ des femmes ayant suivi des études supérieures).

La demande de main-d'oeuvre a également diminué en raison de la crise économique, en particulier dans les secteurs à forte intensité de main-d'œuvre qui sont le plus exposés à la délocalisation de la production dans les pays à bas salaires. Dans le même temps, les réformes des retraites ont prolongé l'âge de la retraite des femmes et des hommes pour atteindre 67 ans, en $2019^{1}$.

1. Loi $n^{\circ} 92$ du 28 juin 2012, la réforme Fornero du régime des retraites a changé radicalement les condi- 
De plus, il convient aussi de prendre en compte l'impact des récentes réformes du travail et des amortisseurs sociaux sur les travailleuses âgées. Les réformes de 2012 et 2015 ont profondément modifié le système, le rendant universaliste - avec une couverture plus large en cas de perte d'emploi et des traitements de base plus généreux axés sur l'historique des cotisations passées et non sur les autres caractéristiques du travailleur ou de l'entreprise (Giorgi, 2018). Toutefois, la Nuova Assicurazione Sociale per l'Impiego (NASpI) réduit d'un an la couverture des revenus qui était garantie aux travailleurs âgés de plus de 50, soit l'allocation de mobilité, versée pendant trois ans après la perte de leur emploi. Par conséquent, les femmes âgées de plus de 50 ans, peu instruites, risquent de connaître une instabilité de revenu jusqu'à la retraite, tandis que le montant de leur retraite sera considérablement réduit.

Les données d'Eurostat (EU-SILC 2019) montrent que $65 \%$ des femmes italiennes au chômage, âgées de 50 à 64 ans, sont menacées de pauvreté ou d'exclusion sociale $(61,2$ \% dans l'UE) ${ }^{3}$. L'asymétrie dans la division du travail domestique et du care entre hommes et femmes ${ }^{4}$ est avérée. Conjuguée à l'insuffisance de la demande de main-d'œuvre dans les secteurs industriels occupant majoritairement la main-d'œuvre féminine, elle peut conduire nombre de femmes de plus de 50 ans au chômage à opérer un arbitrage entre la recherche d'un nouvel emploi régulier sur le marché du travail (avec un possible soutien pour le travail domestique et du care) et la recherche d'un emploi occasionnel ou travail au noir afin de concilier le besoin d'un revenu, bien que modeste, et le rôle de caregiver (prodiguant soins et aide aux proches) dans la famille.

De nombreuses analyses de la condition des travailleurs après la crise de 2008 (Ambrosini \& al., 2014 ; Reyneri \& Fullin, 2013) ont été menées ; cependant, il n’existe pas d'analyse de la condition des femmes âgées et peu qualifiées, ni des tensions qui marquent la transition chômage-emploi des travailleuses âgées. Leur condition après la perte involontaire d'emploi, les stratégies mises en œuvre et les obstacles rencontrés par ces femmes lors de leur réinsertion sur le marché du travail sont encore largement ignorés. L'analyse proposée ici vise à combler, même partiellement, cette lacune.

tions du départ à la retraite. En ce qui concerne la pension de vieillesse, jusqu'en 2011, il était possible de l'obtenir avec 20 ans de cotisations, mais avec 65 ans pour les hommes, 60 ans pour les femmes dans le secteur privé et 61 ans pour les femmes dans le secteur public (système rétributif) ou au moins 35 ans de cotisations versées, 60 ans pour les salariés et 61 ans pour les indépendants (système contributif). Depuis 2012, l'âge de départ à la retraite a progressivement augmenté et atteint actuellement environ 67 ans pour les hommes comme pour les femmes.

2. La nouvelle assurance sociale pour l'emploi est une allocation de chômage mensuelle, définie par l'article 1 er du décret législatif $\mathrm{n}^{\circ} 22$ du 4 mars 2015 .

3. Il s'agit d'une estimation. Cf. Données concernant la population menacée de pauvreté ou d'exclusion sociale (EU-SILC).

4. Selon les données ISTAT (Tempi della vita quotidiana 2016), en 2014, le travail familial (domestique et du care) représentait $21,7 \%$ de la journée moyenne des femmes (5h13), contre 7,6 \% pour les hommes (1h50). 
L'article présente l'analyse de la condition des femmes âgées de 50 ans et plus, peu instruites, ayant perdu leur emploi dans l'industrie du textile, de l'habillement et de la chaussure, en Vénétie et Émilie-Romagne, au cours des dix dernières années.

Il illustre les politiques du marché du travail dans les deux régions et leur efficacité pour cette partie spécifique de la population active. Cette analyse s'enrichit d'entretiens auprès de travailleuses âgées, de partenaires sociaux, de représentants politiques et de fonctionnaires. La comparaison entre la Vénétie et l'Émilie-Romagne vise à mettre en évidence les différences et les similitudes dans les politiques de formation et d'emploi et leurs effets pour les femmes âgées concernées par les restructurations et les licenciements.

\section{Encadré 1. L'enquête}

Cet article est le résultat d'une recherche post-doctorale sur les mutations industrielles, les conditions de travail et les vulnérabilités sociales en temps de crise, visant à analyser la situation des ouvrières peu qualifiées, licenciées par deux entreprises italiennes du textile, de l'habillement et de la chaussure dans deux régions (Vénétie et Émilie-Romagne). À cet effet, nous avons mené cinquante entretiens avec des travailleuses, douze entretiens avec des syndicalistes, six entretiens avec des représentants d'employeurs et six entretiens avec des représentants politiques et fonctionnaires.

Les travailleuses sélectionnées étaient des femmes licenciées de plus de 50 ans, et cherchant un nouvel emploi, réemployées dans une autre entreprise du même secteur ou dans un autre secteur, ou sans travail rémunéré, ou encore effectuant un travail de care non rémunéré au domicile.

Les entretiens avec les travailleuses visaient à examiner leurs trajectoires biographiques et professionnelles, les interruptions de travail, les transitions en cours d'emploi, les interruptions de carrière, les ajustements pour concilier travail et vie personnelle, les conditions de travail, la formation au travail, les effets de la restructuration des entreprises sur la discontinuité du travail, les ajustements du revenu après le licenciement, l'état psychologique lié à la perte d'emploi, la vie quotidienne après le licenciement, la recherche d'un nouvel emploi, les relations avec les collègues, les employeurs et les syndicats, et les effets des réformes des retraites et du marché du travail sur leur vie.

Des entretiens avec des syndicalistes et des représentants d'employeurs ont été menés pour analyser les questions suivantes dans les industries du textile, de l'habillement et de la chaussure : composition de la main-d'œuvre, structure des entreprises, dans une perspective diachronique ; caractéristiques de l'activité de négociation dans ce secteur, effets multiples de la crise économique dans ce secteur, division sexuelle du travail et ses conséquences pour les travailleurs, réformes des amortisseurs sociaux et leur efficacité sur la protection des travailleurs, effets des réformes des retraites et du marché du travail, services et pratiques de reclassement des employés et vieillissement de la main-d'œuvre.

Les entretiens avec les représentants politiques et les fonctionnaires ont porté sur des questions relatives à l'analyse du marché du travail régional, aux politiques du marché du travail mises en œuvre, à la situation des entreprises du textile, du vêtement et de la chaussure, aux limites et au potentiel des politiques publiques, au vieillissement de la main-d'œuvre.

Les régions analysées ont été les suivantes : Valdagno (Vicenza), la province de Trévise, Padoue en Vénétie, Carpi (Modène), S. Mauro Pascoli (RN) et la province de Bologne en Émilie-Romagne. 


\section{Le vieillissement actif face aux restructurations d'entreprises}

L'objet ici est de saisir comment se déclinent les politiques du travail en Vénétie et Émilie-Romagne. Compte tenu de l'homogénéité de la structure productive entre les deux régions, les deux sous-cultures politiques, la blanche (Vénétie) et la rouge (ÉmilieRomagne) ont-elles produit une culture différente en termes de politiques du travail ?

\subsection{Vénétie et Émilie-Romagne : des systèmes de production similaires aux sous-cultures politiques différentes}

Nous examinerons ici, en particulier, la mise en œuvre des politiques du marché du travail après le début de la crise économique, en 2008, et leurs effets sur les femmes licenciées par des entreprises du secteur de la mode dans les régions de Vénétie et d'Émilie-Romagne. Dans ces deux régions, le secteur de la mode, concernant des produits comme la lunetterie, les textiles, les vêtements et les chaussures, a fortement contribué à la croissance de l'économie locale après la Seconde Guerre mondiale et constitue une industrie capitale pour la richesse régionale.

Les régions de Vénétie et d'Émilie-Romagne ont une structure économique similaire. Y est présent un système industriel principalement composé de petites entreprises, axé sur "l'industrie légère ", comme la mode, les meubles et les pièces et composants métalliques. Cette industrie nécessite de faibles quantités de matières premières, d'espace et d'énergie. Le processus de production comporte de nombreuses petites étapes qui peuvent être facilement segmentées, externalisées et délocalisées. Depuis la fin des années 1980, alors que l'Émilie-Romagne a multiplié les activités tertiaires, notamment dans le tourisme et les services aux entreprises, la Vénétie a renforcé sa vocation manufacturière.

Dans les deux régions, l'hégémonie des petites entreprises continue de provoquer des dilemmes, principalement liés à l'innovation économique. Comme le souligne Trigilia (1986, p. 173), "des contraintes d'échelle impliquent que l'économie locale devienne plus dépendante du fonctionnement efficace du système politique régional ». Cependant, le rôle du système politique régional a été très hétérogène dans les régions de la "Troisième Italie $^{5}$ ». À l'inverse, celui des banques locales a été déterminant pour l'économie locale dans presque toutes les régions. Ce qui differe vraiment, c'est le rôle de l'acteur public dans la gouvernance locale.

5. La "Troisième Italie " comprend les régions de Triveneto (Trentin-Haut-Adige, Vénétie, Frioul Vénétie Julienne), les régions " rouges " traditionnelles (Émilie-Romagne, Toscane et Ombrie) et les Marches. Voir Bagnasco (1977). 
En Émilie-Romagne, les institutions ont assuré des taux élevés de dépenses publiques au niveau local et un contrôle permanent du développement socio-économique. En Vénétie, les institutions ont préféré éviter d'intervenir directement dans la sphère économique. Pour expliquer cette différence, Bagnasco \& Oberti (1998, p. 119) insistent sur le fait que, même si le régionalisme de la Vénétie était mitigé lorsque la démocratie chrétienne est parvenue au pouvoir au niveau central, l'hégémonie communiste en Émilie-Romagne, même dans toutes les régions rouges, "constituait un élément fondamental de la force et de la stratégie de ce parti exclu du pouvoir». Au point que, malgré "l'absence de régions réelles et les tendances étatistes de la tradition communiste orthodoxe, le PCI (parti communiste italien) a joué le rôle le plus actif dans la création de ces institutions". Comme le confirment les données récentes sur le montant des dépenses publiques régionales à long terme, l'écart entre l'Emilie-Romagne et la Vénétie est encore considérable ${ }^{6}$. Cependant, une analyse plus approfondie de la composition des dépenses publiques dans les deux régions est nécessaire pour comprendre la dynamique régionale, notamment s'agissant des questions socio-économiques. Les données sur les dépenses régionalisées fournies par le ministère des Finances (2017) montrent en particulier ces principales divisions :

1) Recherche et innovation : après la crise économique, les deux gouvernements régionaux ont réduit leurs dépenses en $\mathrm{R} \& \mathrm{I}$, mais en Vénétie $(-47,15 \%)$ plus qu’en Émilie-Romagne (- 33,44\%). En tout cas, l'investissement régional en R \& I en ÉmilieRomagne est plus élevé qu'en Vénétie ${ }^{7}$;

2) Système d'éducation et de formation : alors que la Vénétie investit davantage dans l'enseignement primaire (en raison également de la forte présence d'écoles catholiques), l'Émilie-Romagne se centre sur l'enseignement supérieur et de troisième cycle. Comme confirmé lors des entretiens avec les responsables politiques, l'Émilie-Romagne a une longue tradition de politiques de formation définies et coordonnées au niveau régional.

La croissance des dépenses en matière de politique du marché du travail est principalement due au fait que les régions italiennes, surtout après le début de la crise économique, ont géré un amortisseur social spécial, la Cassa integrazione in deroga, littéralement " caisse d'intégration des gains en dérogation " (voir l'article de Bisignano, dans ce numéro) Le gouvernement italien a introduit cette mesure spéciale en 2008, afin de garantir un complément de revenu aux travailleurs employés dans des entreprises où les mesures standards sont absentes, ou ne percevant plus d'indemnités ${ }^{8}$.

6. Cf. Les données sur les dépenses courantes, notamment sur les transferts aux administrations publiques opérés par le ministère de l'Économie et des Finances sur les dépenses de l'État régional (en italien, estimations provisoires), sont disponibles à l'adresse suivante : http://www.rgs.mef.gov.it/VERSION-I/pubblicazioni/ pubblicazioni_statistiche/la_spesa_statale_regionalizzata/index.html [Consulté le 15 avril 2018].

7. En 2008, les fonds alloués à la recherche et à l'innovation, en Vénétie, se sont élevés à 29130 euros (37 154 en Émilie-Romagne), en 2015, ils sont tombés à 15397 euros en Vénétie et à 24731 euros en Émilie-Romagne.

8. Il s'agit d'une indemnité mensuelle pour les travailleurs mis à pied temporairement par des entreprises 
Jusqu’en 2014, il y avait aussi la Mobilità in deroga, littéralement «indemnité de réaffectation ou de mobilité par dérogation », qui aidait les travailleurs à rechercher un nouvel emploi, comme c'était le cas pour l'allocation de mobilité traditionnelle. La mise en ouvre de cette politique du marché du travail est prévue par un accord entre l'État et les régions : l'État finance les compléments de revenu, tandis que les fonds régionaux financent les actions des politiques actives du marché du travail. Cet accord comporte des négociations avec les syndicats au niveau régional. La Région doit ensuite examiner et approuver les demandes des entreprises, puis autoriser l'Institut national de la sécurité sociale (INPS) à verser les prestations à chaque travailleur non salarié. Les employeurs ne financent pas cette politique spéciale du travail (comme le prévoit la loi sur les amortisseurs sociaux standard comme l'Indennità di mobilità $)^{9}$. Le financement provient du Fonds social européen (FSE) et la mesure de soutien des salaires est étroitement liée à des politiques actives du marché du travail, notamment des politiques de formation et d'emploi, assurant un équilibre entre les politiques passives et actives. À cet égard, la région ÉmilieRomagne a accru l'investissement public dans la formation professionnelle, combiné à un système de formalisation et de certification des compétences, pour former une maind'œuvre qualifiée ; la Vénétie a fortement investi dans le renforcement de la compétitivité des entreprises (principalement pour promouvoir sa vocation d'exportation) et moins dans la formation et l'amélioration des compétences des travailleurs. En 2019, l'INPS a révisé les dispositions actuelles concernant l'octroi de cette indemnité en dérogation, pour une nouvelle période de douze mois, pour les régions en crise industrielle complexe ${ }^{10}$. Les amortisseurs en dérogation - Cassa integrazione in deroga e mobilità in deroga - étaient destinés aux secteurs de production non couverts par les protections " ordinaires " de soutien du revenu. Cependant, afin d'accroître la protection face aux crises, ils ont souvent été utilisés dans des secteurs déjà couverts par des amortisseurs ordinaires, mais qui les avaient en fait épuisés. Toutefois, soulignons que depuis leur création, ces mesures ont répondu davantage aux besoins locaux qu'à la nécessité d'une réforme intégrale des politiques du marché du travail national.

Les tendances de l'emploi des femmes suivent les sous-cultures politiques profondément enracinées qui caractérisent ces deux régions. En 1977, l'écart entre le taux d'emploi

(principalement des entreprises artisanales et des petites entreprises industrielles de moins de quinze employés). Voir loi n ${ }^{\circ}$ 203/2008, loi n²/2009, loi n 33/2009, loi n 92/2012 et la loi de stabilité de 2016.

9. Littéralement « indemnité de réaffectation ou de mobilité ». Le montant de l'indemnité équivalait à celui de l'indemnité de licenciement pendant la première année et à $80 \%$ de cette allocation ensuite ; l'indemnité pouvait être perçue à l'avance et en un seul versement par les travailleurs souhaitant démarrer une activité professionnelle indépendante ou en association. Cette indemnité a été supprimée lors de la dernière réforme du système de sécurité sociale en 2015.

10. Le Decreto crescita (Décret de croissance) prévoyait en effet l'extension aux travailleurs ayant cessé ou cessant leur mobilité ordinaire ou par dérogation au 31 décembre 2019, dans la limite de 16 millions d'euros pour l'année 2019 et 10 millions d'euros pour 2020. 
des femmes, en Émilie-Romagne et en Vénétie dépassait neuf points de pourcentage ${ }^{11}$. L'écart entre les deux régions semble être important puisque les femmes qui vivent en Émilie-Romagne sont en moyenne plus actives et occupées que celles résidant en Vénétie. Après la crise économique de 2008, même si l'écart s'est considérablement réduit, il persiste : en 2018, le taux d'activité des femmes, âgées de 55 à 64 ans, en Vénétie, était de 47,5\%, contre 55,8 \% en Émilie-Romagne ; le taux d'emploi en Vénétie était de 46,1\%, contre 53,9\% en Émilie-Romagne ${ }^{12}$.

\section{2. Être au chômage et loin de la retraite : politiques régionales et paradoxes du vieillissement actif}

L'industrie de la mode, en particulier après la crise pétrolière des années 1970, a connu de nombreux changements. Cette industrie se caractérise par une discontinuité structurelle dans les temps de travail, due au caractère saisonnier de la production. À partir de la fin des années 1970, les entreprises du secteur du textile, de l'habillement et de la chaussure ont connu des taux de croissance et de contraction variables, au point qu'il est difficile de définir une tendance stable. L'expiration de l'Arrangement multifibres (AMF) en 2005 - qui limitait les exportations de textiles et de vêtements vers les plus grands marchés du monde, États-Unis, Canada et Union européenne (UE), afin de protéger les producteurs des pays industrialisés de leurs concurrents asiatiques a encouragé un vaste processus de restructuration des entreprises et de délocalisation de certains segments de la production dans les pays à bas salaires ${ }^{13}$.

Soulignons que, jusqu'en 2014, l'indemnité de réaffectation ou de mobilité par dérogation, et jusqu'en janvier 2017, l'indemnité de réaffectation ou de mobilité traditionnelle, ont permis à de nombreux travailleurs de retrouver un emploi, grâce à l'attribution d'une subvention aux employeurs qui embauchaient un travailleur issu de la « liste de mobilité ${ }^{14} »$.

11. Voir les séries chronologiques ISTAT sur le marché du travail à l'adresse suivante : http://timeseries. istat.it/.

12. Voir les données ISTAT sur travail et salaires (en italien et anglais) à l'adresse suivante : https://www. istat.it/it/lavoro-e-retribuzioni?dati.

13. Voir Urry (2014).

14. Les listes de mobilité étaient des listes spéciales, établies par la loi 223/1991, dans lesquelles étaient inscrites les personnes licenciées collectivement par les entreprises de plus de quinze salariés en raison de la fin, de la transformation ou de la réduction de l'activité ou du travail. L'article 2 de la loi n ${ }^{\circ} 92$ de 2012 (Réforme Fornero) a instauré, à compter du 1er janvier 2017, l'abrogation des dispositifs liés à la mobilité. En particulier, il s'agissait de l'allocation de mobilité ordinaire, de la possibilité d'inscription sur les listes de mobilité et des avantages qui en résultaient pour le recrutement des travailleurs qui y étaient enregistrés. Jusqu'au 31 décembre 2016, dans le cas d'un emploi permanent à plein temps d'un travailleur figurant sur les listes de mobilité, l'employeur avait droit à des avantages particuliers : 1) pour chaque travailleur inscrit sur la liste de mobilité et embauché pour une durée indéterminée, le taux de cotisation de l'employeur était, pour les 18 premiers mois, celui prévu pour les apprentis $(10 \%) ; 2)$ pour chaque mois de salaire versé au 
La réforme des politiques du marché du travail, lancée avec le Jobs Act, avec en particulier l'introduction de la nouvelle disposition d'assurance sociale pour l'emploi (NASpI), a réduit l'allocation de chômage pour les travailleurs âgés de 50 ans et plus et a supprimé la subvention allouée aux employeurs. Les deux dernières lois de finance ont constitué des incitations à l'embauche (bonus disoccupati) pour les employeurs embauchant (sous contrat à temps plein et à durée indéterminée) des chômeurs de longue durée. Néanmoins, ces incitations sont loin d'accroître les chances des travailleurs de trouver un nouvel emploi, comme l'ont souligné tous les syndicalistes interrogés. Surtout, comme on le verra, s'il agit des femmes âgées.

Quant aux restructurations d'entreprises survenues après 2008, les gouvernements régionaux d'Émilie-Romagne et de Vénétie ont instauré différentes mesures. En 2015, l'Émilie-Romagne a lancé le "Pacte du travail ", signé par la région, les autorités locales, les syndicats, les organisations professionnelles, le forum du troisième secteur ${ }^{15}$, les universités et le bureau régional de l'éducation. Cet accord s'est construit autour d'un programme financé par les ressources du Fonds social européen, dont les coûts opérationnels totaux s'élèveront à 786250182 euros pour six ans (2014/2020).

Le Pacte vise à initier une nouvelle génération de politiques publiques fondées sur diverses actions visant à favoriser : l'accès à l'emploi des chômeurs et des personnes inactives ; l'intégration durable dans le marché du travail des jeunes (Initiative européenne pour la Jeunesse), en particulier des NEET (Not in employement, education and training ou jeunes déscolarisés, sans emploi et ne suivant aucune formation) ; l'égalité entre hommes et femmes; la capacité d'adaptation des travailleurs, des entreprises et des entrepreneurs au changement ; la modernisation des institutions du marché du travail ; l'inclusion active ${ }^{16}$; l'accès à des services de l'emploi abordables, durables et de haute qualité.

Depuis novembre 2017, les personnes au chômage de longue durée ne percevant pas de complément de revenu peuvent choisir de bénéficier des services proposés par le centre pour l'emploi où elles se sont inscrites ou de ceux proposés par l'un des vingt acteurs privés agréés, avec plus de 170 bureaux répartis dans toute la région. Il n’y a pas

travailleur, l'employeur recevait, par le biais du système de solde des cotisations, $50 \%$ de l'allocation de mobilité pendant douze mois ou plus, en fonction de l'âge et du lieu de résidence (12 mois pour les travailleurs jusqu'à 50 ans ; 24 mois pour les travailleurs de plus de 50 ans ; 36 mois pour les zones du Sud ou dans les zones désavantagées).

15. Le Troisième secteur rassemble une multiplicité d'acteurs (associations, fondations, organisations nongouvernementales, organisations religieuses etc.) intervenant dans la sphère publique, et dont les traits communs sont le bénévolat et le caractère non lucratif de l'activité.

16. Selon la Commission européenne, l'inclusion active consiste à permettre à chaque citoyen de participer pleinement à la société, et notamment d'exercer un emploi. Pour atteindre cet objectif, les gouvernements européens doivent soutenir : une aide au revenu adéquate; des marchés du travail ouverts à tous ; un accès à des services de qualité qui aident les citoyens à participer activement à la société, et notamment à revenir sur le marché du travail. Voir : https://ec.europa.eu/social/main.jsp?catId=1059\&langId=fr 
de politiques ciblées pour les plus de 50 ans. Cependant, en 2015, la région a défini l'indice de fragilité, une mesure visant à rapprocher les services professionnels, sociaux et de santé pour les personnes en situation de fragilité et de vulnérabilité, comme prévu par la loi régionale $n^{\circ} 14 / 2015$. Cette loi soutient la réinsertion professionnelle et sociale des personnes en situation de fragilité et de vulnérabilité "par l'intégration des services publics de l'emploi, sociaux et de santé, en tant que réponse unitaire au problème des conditions multi-problématiques qui caractérisent les personnes fragiles et vulnérables".

Depuis 2012, la Vénétie a pris des mesures concernant la réinsertion professionnelle des chômeurs de 50 ans et plus. En 2012, le premier accord a été signé entre la Région, la Fondazione Cassa di Risparmio di Padova e Rovigo ${ }^{17}$ et Etra (société multi-services entièrement publique). Cet accord a financé des dispositifs de réinsertion des chômeurs et chômeuses de plus de 50 ans. ${ }^{18}$ Près de 30 millions d'euros ont été alloués à ces projets, impliquant 1000 chômeurs/chômeuses ayant participé à des activités de reconversion destinées au placement et au suivi de stages. D’une durée maximale de quatre mois, ces stages obligent les participants à suivre une formation dans des entreprises locales. En 2017, la Vénétie a signé un nouvel accord au niveau local ${ }^{19}$ afin de mettre en œuvre des mesures actives de politique de l'emploi pour les personnes touchées par les restructurations d'entreprises. Cet accord a été suivi par un appel lancé par la Vénétie, en 2018, pour la mise en œuvre de politiques actives du marché du travail pour ces mêmes travailleurs. Dans les deux cas, aucune distinction n’a été opérée sur la base du sexe des travailleurs concernés. Compte tenu des mesures prises au niveau régional, un écart apparaît entre les politiques du marché du travail adoptées dans les deux régions. En Émilie-Romagne, il y a une coordination centrale des politiques du marché du travail : la formation professionnelle, les politiques du travail actives et passives sont conçues pour toute la région. Le « Pacte du travail », signé en 2015, peut être considéré comme le résultat d'un large débat entre la Région, les partenaires sociaux, les autorités locales, le forum du troisième secteur et les universités. En revanche, en Vénétie, les initiatives lancées ces dernières années ont été annoncées lors des délibérations du conseil régional, sans négociations avec les partenaires sociaux.

L'efficacité des deux stratégies n'a pas encore été évaluée. Les syndicalistes et les représentants des employeurs interrogés dans les deux régions sont conscients du problème de la réinsertion des travailleurs âgés, notamment des femmes âgées de 50 ans et plus et précédemment employées dans le secteur de la mode, mais ils sont

17. La Fondazione Cassa di Risparmio di Padova e Rovigo a été fondée en 1991. Elle finance des projets destinés au développement économique, social et culturel. Les sociétés coopératives et les entreprises sociales participent aux appels à propositions lancés par la Fondation pour activer des projets au niveau local. Il n'existe pas d'instrument relatif à l'évaluation des résultats produits par ces projets.

18. Il concernait 44 municipalités de la province de Padoue, 30 de la province de Vicence et une de la province de Trévise.

19. Il concernait 74 municipalités de la province de Vérone et 112 de la province de Vicence. 
également conscients que les politiques, dans ce domaine, sont loin d'être conçues pour les travailleuses âgées, en raison des restructurations en cours dans ce secteur et de la pénurie de nouveaux emplois disponibles.

\section{Les travailleuses face à la crise : une recherche d'emploi difficile}

Les femmes interviewées ont un faible niveau d'instruction, principalement en raison de la seule fréquentation de la scolarité obligatoire, et ont parfois aussi une ancienneté élevée dans l'industrie de la mode. Elles ont toutes été licenciées après 2008. Au moment de l'entretien, elles travaillaient déjà dans une autre entreprise du même secteur ou dans un autre secteur, ou bien elles étaient encore à la recherche d'un emploi stable. Le report de l'âge de la retraite à 67 ans (en 2019) les contraint à trouver un emploi permanent, afin d'éviter la baisse du montant de la pension de retraite. Cependant, les compétences acquises dans l'industrie de la mode ne sont pas facilement utilisables dans un autre secteur et l'absence ou l'insuffisance de politiques actives du travail destinées aux seniors ne facilite pas leur recherche d'emploi. Comment les travailleuses interviewées font-elles face à la recherche d'un nouvel emploi ? Un premier niveau d'analyse consistera à examiner leur rapport à la formation au cours de leur carrière et après la perte de leur emploi. Un second niveau d'analyse attestera des difficultés rencontrées dans la recherche d'un nouvel emploi, notamment liées aux obstacles organisationnels et socio-culturels à la conciliation vie professionnelle-vie familiale.

\subsection{Un faible recours à la formation}

En Italie, surtout au cours des dernières décennies, la formation professionnelle a été largement redéfinie. Toutefois, la réglementation principale fait encore référence à la loicadre sur la formation professionnelle $\mathrm{n}^{\circ}$ 845, du 21 décembre 1978. Cette loi souligne le rôle de la République italienne dans la promotion de "la formation et l'amélioration professionnelle du citoyen dans la mise en xuvre des articles 3, 4, 35 et 38 de la Constitution, afin de rendre effectif le droit de travailler et de le choisir librement, et de favoriser la croissance des travailleurs "; tout en étant considérée comme un instrument d'employabilité, la formation professionnelle vise " à favoriser l'emploi, la production et l'évolution de l'organisation du travail en fonction des progrès scientifiques et technologiques " (art. 1).

En outre, les initiatives de formation professionnelle sont considérées comme "un service d'intérêt général destiné à assurer un système d'actions de formation visant à diffuser les connaissances théoriques et pratiques nécessaires à l'exercice de métiers professionnels et à se concentrer sur la primo-insertion, la formation, la spécialisation, la formation continue des travailleurs dans un cadre d'apprentissage tout au long de la vie " (art 2). 
Selon une étude sur la formation professionnelle continue (ISFOL 2015), en 2014, le taux de participation de la population adulte (25 à 64 ans) aux activités d'éducation et de formation atteignait $8 \%$ en Italie, contre 18,3\% en France et 10,7 \% en UE. Les résultats de l'enquête ont souligné que la santé et la sécurité sur le lieu de travail est le thème le plus récurrent de la formation professionnelle menée en Italie. De même, parmi les participants, la proportion de ceux qui bénéficient d'un contrat à durée indéterminée et qui occupent des postes à haut niveau de responsabilité dans l'entreprise est la plus élevée.

Les travailleuses enquêtées au cours de la recherche menée dans les deux régions ont une ancienneté moyenne élevée dans le même secteur. S'agissant de la formation professionnelle, elles affirment que depuis le premier emploi rémunéré jusqu'au licenciement, elles n'ont participé à aucune action de formation pour améliorer leurs compétences techniques, ni aux sessions de formation destinées à faire face aux changements technologiques et numériques.

Roberta (53 ans, Émilie-Romagne, mariée, trois enfants âgés de 17, 21 et 25 ans) souligne qu'en l'absence d'initiatives de formation émanant de l'entreprise, les contraintes liées au travail du care empêchent de se consacrer à une formation personnelle en dehors des heures de travail, avec toutes les conséquences négatives que cela entraîne :

"J'ai changé cinq fois d'emploi, mais je n'ai jamais suivi de formation pendant mon travail. L'entreprise a uniquement dispensé une formation en matière de sécurité (obligatoire ...). Je n'ai pas eu le temps de me réorienter professionnellement. Au cours des vingt dernières années, jai eu trois enfants. Il n'y avait guère de temps pour se former en dehors des heures de travail. J'ai dîu travailler à temps partiel pour élever mes enfants. Ils sont toujours avec moi, même sills ont un père..."

Les changements intervenus dans les processus de production du textile, de l'habillement et de la chaussure ont induit une polarisation des emplois. Elles s'est traduite par une fragmentation des tâches pour les travailleurs moins qualifiés occupant des emplois à haute intensité de main-d'œuvre. La formation est particulièrement importante pour les travailleurs âgés, dont les compétences acquises au travail, tout au long de l'apprentissage pratique, risquent de devenir rapidement obsolètes, ce qui rend ces travailleurs parfaitement remplaçables (Lee \& al., 2008 ; Behaghel \& al., 2014 ; Williams van Rooij 2014). De plus, le manque de formation des travailleurs peu scolarisés augmente le risque d'exclusion sociale, surtout pour les femmes. La discontinuité des carrières féminines, en raison de la surcharge liée au travail du care, freine la reconversion professionnelle des travailleuses.

Anna (52 ans, Vénétie, mariée, deux enfants âgés de 20 et 22 ans), lorsqu’elle présente sa situation, explique :

"Ce n'est pas facile quand on perd l'emploi à cinquante ans. Maintenant, comme je ne travaille plus, je vais devoir prendre ma retraite avec une pension très basse. Les femmes sont très discriminées, surtout à mon âge. Nous ne sommes plus des travailleuses, nous sommes des femmes de cinquante ans. Les entrepreneurs ne nous considèrent pas comme très vigoureuses. Ils croient que 
nous avons moins de capacités cognitives que les jeunes. Il est évident que l'àge est crucial autant que l'expérience. J'en ai vraiment marre de chercher un emploi!"

Quant aux employées de plus de cinquante ans, la discrimination se nourrit de l'apport des caractéristiques généralement attribuées aux travailleurs âgés : difficultés d'adaptation aux innovations technologiques et capacité limitée de résistance à l'effort physique (Drury, 1994 ; West \& al., 2015). Plusieurs recherches (Duncan \& Loretto, 2004 ; Moore, 2009) ont montré que souvent, en raison de stéréotypes sexistes profondément enracinés, les personnes âgées de plus de cinquante ans considéraient elles-mêmes l'âge non seulement comme un obstacle réel à l'entrée ou à la réintégration dans le système d'emploi, mais également comme un obstacle à la formation et à la progression de carrière.

Sandra (54 ans, Vénétie) ne croit pas en la possibilité de réorienter son parcours professionnel :

" $\grave{A}$ mon âge, je ne sais pas si je suis encore capable d'apprendre de nouvelles choses. Je préférerais trouver un autre emploi dans mon propre domaine. Si je ne peux pas le trouver... franchement... je ne veux pas commencer à suivre des cours."

Compte tenu des limites imposées par l'État aux activités de formation en cours d'emploi, la contribution des entreprises devient cruciale (Streeck, 1989) ${ }^{20}$. De cette manière, la petite taille des entreprises des deux régions analysées et le faible investissement technologique dans le processus de production limitent grandement cette contribution (ISFOL, 2013).

\subsection{La conciliation vie professionnelle/vie privée}

Les travailleuses interviewées sont principalement des femmes mariées ou vivant en couple avec des enfants. Le régime de protection sociale italien a longtemps été marqué par l'hégémonie du modèle classique du soutien de famille masculin. Il en résultait une grande asymétrie entre les sexes dans le travail rémunéré et non rémunéré. En termes de division du travail domestique et du care, un double scénario se dégage : parmi les femmes interviewées, il y a celles qui ont toujours effectué toutes seules le travail domestique et du care et d'autres qui le partagent avec leurs conjoints. À cet égard, il faut souligner qu'au cours de leur carrière professionnelle, pour toutes les femmes interviewées, la rigidité de l'organisation du travail rémunéré a empêché une flexibilité positive permettant de concilier vie professionnelle et vie privée.

20. L'investissement dans la formation pour l'augmentation et la diversification des compétences des travailleurs-euses est essentiel dans les entreprises dont l'objectif est l'innovation et la qualité de la production. Streeck souligne que la formation est un bien public et que l'investissement dans la formation est la garantie la plus puissante pour maintenir et augmenter les salaires dans la nouvelle division internationale du travail qui a été mise en place après la fin des Trente Glorieuses. 
Norma (53 ans, Émilie-Romagne, mariée, deux enfants âgés de 19 et 23 ans qui vivent en famille) a souvent changé d'emploi pour s'occuper de ses enfants :

"J'ai toujours cherché un emploi à temps partiel pour gérer les enfants. J'ai occupé beaucoup d'emplois afin d'avoir une flexibilité horaire qui me permettait de m'occuper des enfants. Mon fils, âgé de sept ans, avait une tumeur maligne du rein et a suivi treize mois de chimiothérapie et de radiothérapie et bien évidemment, je ne pouvais pas travailler. J'ai recommencé à travailler quand mon fils a été en meilleure santé, dans une boulangerie locale, toujours à temps partiel. J'ai ensuite trouvé un emploi dans une usine de chaussures pendant dix-sept ans, puis j'ai été licenciée."

La rigidité du temps de travail et le manque de politiques en termes de conciliation vie professionnelle/vie privée contraignent souvent les femmes à rechercher du travail au noir, compte tenu de la forte proportion de travailleurs concernés par l'économie souterraine. À cet égard, Diana (56 ans, Émilie-Romagne, divorcée, vit seule) déclare :

"J'ai toujours eu des emplois irréguliers et discontinus. C'était plus pratique pour moi de travailler au noir pour élever mes deux filles. Bien sûr, c'était très rentable pour les entreprises aussi. Pour moi, c'était la seule chance de continuer à travailler. Le travail à temps partiel dans le secteur du tricot faisait tout simplement défaut. J'ai fait du travail au noir pendant dix ans et maintenant je suis pénalisée par rapport au montant de ma pension de retraite."

Avant de perdre leur emploi, le fardeau du travail domestique et du care reposait sur les épaules de la plupart des femmes interviewées, avec le soutien de la famille, sans réduction des heures de travail. Cependant, dans l'industrie du textile, de l'habillement et de la chaussure, même si la majorité des employés sont des femmes, la réduction du temps de travail a toujours été problématique.

Amelia (52 ans, Émilie-Romagne, mariée, deux enfants de 17 et 22 ans à charge) aimerait travailler à temps partiel :

"Je cherche un travail à temps partiel, mais ce n'est pas facile. J'ai toujours demandé à faire un travail à temps partiel pour moccuper des enfants et de la famille. Dans la famille, aussi dans ma famille, les rôles sont toujours très stricts. La fermme reste la principale référence pour les enfants et le travail domestique. Le mari peut seulement aider... Je ne pense pas que nous allons vers une transformation radicale de la situation actuelle. Mes fils font du travail domestique, mais ils voient l'exemple à la maison : la mère est toujours présente, le père n'est jamais là ..."

D'autre part, le travail à temps partiel s'avère souvent un piège pour les travailleuses qui en font l'expérience (Maruani, 1996 ; Angeloff, 1999 ; Fuchs Epstein \& al., 1999)21. Surtout dans l'industrie, car dans la grande distribution, la plupart des emplois de bas niveaux de

21. Le travail à temps partiel implique de renoncer aux revenus actuels, mais aussi futurs (montant de la pension). De plus, puisque l'avancement professionnel est souvent lié au temps passé sur le lieu de travail, le travail à temps partiel a des conséquentes importantes qui risquent plus d'accroître les discriminations liées au genre que de les réduire. 
qualification sont à temps partiel (subi). En revanche, la recherche d'un nouvel emploi est très difficile, comme le souligne Luisa (52 ans, Émilie-Romagne, mariée, trois enfants âgés de 13,15 et 18 ans) :

"Je n'ai pas eu la chance d'avoir des parents pour prendre en charge mes enfants, alors je devais men occuper toute seule. Pendant des années, au déjeuner, je devais gérer mes trois enfants. Dans l'après-midi, je devais les emmener à des activités sportives. Je n'avais que deux heures dans l'après-midi pour faire le ménage. Pendant dix ans, la possibilité de concilier travail et vie privée a été une bonne chose au travail. À la direction de l'entreprise, il y avait une femme très compréhensive. Mais ensuite, la direction a changé et j'ai été licenciée. Pendant des années, jai travaillé plus que mon temps de travail contractuel, mais cela ne m'a pas été utile. Si on travaille à temps partiel, on est moins protégé. Maintenant, après le licenciement, je cherche un emploi à temps partiel de six heures, mais il est impossible de le trouver. Pour les employeurs, le temps partiel présente de nombreuses difficultés de gestion."

Après le licenciement, la répartition du travail domestique et du care, au sein du couple, a perduré, sans aucun changement significatif. Les femmes interrogées en Émilie-Romagne ont des compagnons plus impliqués dans le partage des tâches domestiques et du care. Néanmoins, le chômage, s'il se prolonge, redéfinit ces équilibres. Pour la plupart des femmes interviewées, notamment les plus âgées, un arbitrage s'opère au sein du ménage, entre travail discontinu et travail non rémunéré : elles ne trouvent aucun travail rémunéré, tandis que dans la famille, le travail du care augmente. Il n'y a plus de jeunes enfants à élever, mais pour beaucoup d'entre elles, il y a des parents âgés et des petits-enfants. Par conséquent, si l'âge de la retraite n'est pas très éloigné, elles décident de se consacrer au travail non rémunéré à la maison, plutôt que de chercher un travail hypothétique.

\subsection{Après le licenciement, une recherche d'emploi marquée par la pénurie d'emplois}

Les travailleuses interviewées ne considèrent pas les services publics de l'emploi comme efficaces pour trouver un nouvel emploi. Les discriminations persistantes à l'encontre des travailleuses âgées découragent la recherche d'emploi. Il a été prouvé que la double discrimination à l'égard des travailleuses âgées, fondée sur des stéréotypes liés au sexe et à l'âge, est souvent liée à leur plus grande vulnérabilité sur le marché du travail, en raison de trajectoires de travail spécifiques aux femmes (interruptions de carrière, travail à temps partiel ou occasionnel et écart de rémunération) ${ }^{22}$. De plus, l'absence de politiques actives du marché du travail ciblées engendre des risques élevés de pauvreté pour les femmes âgées (Corsi \& al., 2010).

Le manque d'opportunités pour les travailleuses âgées est lié non seulement au déséquilibre structurel entre la demande et l'offre de main-d'œuvre, mais également au

22. Voir Duncan \& Loretto (2004), Loretto \& al. (2007). 
manque de création d'emplois. Soulignons que dans les pays occidentaux, le vieillissement actif, surtout par rapport aux femmes, n’est pas récent dans le débat politique (Arber \& Ginn, 1991 ; Gist \& Velkoff, 1997). Néanmoins, au cours des dernières décennies, la principale réponse politique au vieillissement de la population a été de réformer les systèmes de sécurité sociale afin de réduire les dépenses publiques consacrées aux retraites, plutôt que d'investir dans des politiques d'apprentissage tout au long de la vie et de conciliation vie professionnelle-vie privée.

Les entretiens ont clairement mis en évidence la tension qui existe entre la pression exercée sur les travailleurs de plus de 50 ans pour qu'elles se réinsèrent rapidement sur le marché du travail et les limites objectives liées à la pénurie d'emplois.

Luisa ( 55 ans, Vénétie, mariée, une fille âgée de 27 ans et dont les parents ont des besoins en termes de care) souligne cette tension et le paradoxe qui émerge :

"Au niveau national, ni les syndicats ni les partis politiques n'ont agi pour limiter l'exode des entreprises italiennes à l'étranger. Cette délocalisation sauvage a pénalisé des milliers de travailleurs. Perdre son emploi à cinquante ans, avec cette nouvelle réforme des retraites... Si j'avais continué à travailler, j'aurais pu prendre ma retraite d'ici 2022. Maintenant, je ne sais pas quand je prendrai ma retraite. Pour ceux qui ont commencé à travailler tôt, la situation est très difficile. Les employeurs pensent qu'une femme de cinquante ans a moins de force physique et moins de capacités cognitives qu'une jeune femme. Je pense que c'est un stéréotype. Si notre politique nous dit que nous devons prendre notre retraite plus tard et toujours plus tard, cela signifie que nous avons la capacité de rester au travail, mais c'est le travail qui nous manque."

Pour les femmes interviewées, le manque d'éducation formelle et de formation professionnelle rend difficile la recherche d'un emploi dans un autre secteur économique. Les services pour l'emploi offrent des cours d'informatique et des formations pour améliorer les compétences de base en matière de recherche d'emploi, mais cela ne suffit pas, comme nous l'explique Giovanna (51 ans, Vénétie, mariée, deux enfants de 17 et 20 ans à charge) :

"J'ai suivi des cours de formation, anglais, cours d'informatique. Je me suis également inscrite dans des agences de travail temporaire. En deux ans, je n'ai reçu aucun appel. Les réponses sont toujours les mêmes : les employeurs recherchent des filles de vingt ans qui viennent de terminer leurs études secondaires. Nous sommes et resteront au chômage!"

Les femmes interviewées qui ont déjà un emploi affirment qu'elles l'ont trouvé via des réseaux informels, certaines faisant du travail au noir, tandis que les femmes totalement au chômage sont découragées. À cet égard, Beatrice (54 ans, Émilie-Romagne, mariée, deux enfants âgés de 27 et 30 ans vivant dans la famille, tout comme sa mère) déclare :

"Après le licenciement, j'ai réussi à obtenir l'indemnité de mobilité. Elle s'élève à 70 euros. Je suis allée au centre pour l'emploi. J'ai suivi un cours d'informatique. C'était utile, mais j'ai travaillé dans une entreprise de tricot pendant trente ans... Dans ce secteur, il 
n'y a aucun moyen de trouver un emploi et je trouve difficile de travailler dans d'autres secteurs."

Les entreprises n'ont pas conçu de mesures d'accompagnement à la réinsertion et à la recherche d'emploi et les politiques publiques ne les concernent pas du tout. Chiara (55 ans, Vénétie, mariée, vit avec son mari. Ella a deux enfants de 35 et 37 ans autonomes) souligne l'inefficacité du contrôle public sur les processus de restructuration des entreprises :

"Pour la première fois, à l'âge de 55 ans, je connais le licenciement! Avant, jai été transférée du bureau à la production. Finalement, le chantage de perdre l'emploi a amené tout le monde à tout faire. J'ai été engagée sur les listes de mobilité, mais lorsque les réductions d'impôts ont pris fin, l'employeur a décidé de me licencier. Je me suis inscrite au centre pour l'emploi... Je doute que là-bas ils trouvent du travail à quelqu'un. Au centre pour l'emploi, les employés soccupent du dossier pour t'assurer la sécurité sociale, mais ils ne font rien d'autre. Les gens ne se tournent pas vers les institutions. Il nont pas confiance. Les entreprises peuvent licencier les travailleurs quand elles le souhaitent. La crise économique a été un grand alibi. Il n'y a pas de contrôle effectif de la situation budgétaire des entreprises. Les gens, même avec beaucoup d'ancienneté dans l'entreprise, sont mis à la porte très facilement. "

Le droit à une procédure de réinsertion professionnelle devrait être accordé à tous les travailleurs licenciés ${ }^{23}$. Cependant, la responsabilité sociale des entreprises dans ce domaine est loin d'être débattue. Ainsi, l'absence historique de politique industrielle compte beaucoup, comme le souligne Anna (52 ans, Vénétie, mariée, une fille de 25 ans indépendante) :

"L'employeur a fait ce qu'il voulait faire, malgré l'opposition des syndicats. Au niveau local, nous avons lutté contre les licenciements. Je pense que le problème est au niveau national. Il y a trente ans, les gouvernements italiens auraient dî empêcher la délocalisation massive qui a affecté ce secteur, avec des politiques spécifiques. Au lieu de cela, ils ont laissé partir des entreprises. "

\section{Conclusion}

L'analyse des études de cas réalisées vise à montrer comment les femmes de 50 ans et plus, licenciées des entreprises du secteur de la mode, et en particulier du textile, de l'habillement et de la chaussure, ont essayé de gérer leur vie après le licenciement. Compte tenu des transformations en cours de cette industrie - qui, depuis la seconde moitié des

23. L'art. 8 de la Convention OIT ( ${ }^{\circ} 168$ ) sur la promotion de l'emploi et la protection contre le chômage (1988) : tout Membre doit s'efforcer d'adopter, sous réserve de la législation et de la pratique nationales, des mesures spéciales pour promouvoir des possibilités additionnelles d'emploi et d'aide à l'emploi et faciliter l'emploi productif et librement choisi de catégories déterminées de personnes désavantagées qui ont ou qui sont susceptibles d'avoir des difficultés à trouver un emploi durable, comme les femmes, les jeunes travailleurs, les personnes handicapées, les travailleurs âgés, les chômeurs de longue durée, les travailleurs migrants en situation régulière et les travailleurs affectés par des changements structuraux. L'art. 29 de la Charte des droits fondamentaux de l'Union européenne (2016) souligne que toute personne a le droit d'accéder à un service gratuit de placement. 
années 1970, n'a cessé d'être, en quelque sorte, en restructuration permanente engendrant la réduction de l'emploi - il convient de comprendre comment les politiques publiques et le dialogue social ont fait face à l'émergence de nouvelles vulnérabilités.

Jusqu'en 2017, les deux gouvernements régionaux de la Vénétie et de l'Emilie Romagne ont géré la restructuration des entreprises principalement par le report des licenciements, avec un recours généralisé aux amortisseurs sociaux dérogatoires (fonds de garantie des salaires et allocation de mobilité). Les réformes intervenues dans le système d'amortisseurs sociaux ont progressivement réduit la marge de manœuvre de cette stratégie. Parallèlement, la réforme des retraites, lancée en 2011, qui a relevé l'âge de départ à la retraite à 67 ans pour les hommes comme pour les femmes, d'ici à 2019, a engendré de nouveaux dilemmes pour les travailleurs âgés, en particulier pour les femmes qui ont des parcours professionnels discontinus et sont encore loin de la retraite.

En conclusion, les restructurations et les licenciements d'entreprises ont été gérés par des institutions régionales et des partenaires sociaux avec des outils traditionnels, principalement des amortisseurs sociaux, destinés à réintégrer les travailleurs licenciés dans le marché du travail.

Toutefois, il n’y a pas eu de politiques actives du marché du travail destinées aux travailleuses âgées. Les femmes de 50 ans et plus, peu qualifiées, encore loin de la retraite, ont besoin de politiques spécifiques efficaces, permettant l'acquisition de nouvelles qualifications. Cette lacune confirme ce que la littérature a mis en évidence jusqu’à présent en termes de discrimination à l'égard des travailleuses âgées (Arber \& Ginn, op. cit. ; Duncan $\&$ Loretto, op. cit. ; Moore, op. cit.). Les différences régionales entre les sous-cultures politiques n'ont aucune incidence sur l'efficacité du soutien à cette partie spécifique de la population active.

L'analyse de ces cas met en lumière certains problèmes clefs : malgré la pression exercée par le vieillissement actif, les femmes âgées sont fortement stigmatisées sur le marché du travail, même dans les régions où leur participation à un travail rémunéré constitue une préoccupation de longue date ; les politiques du marché du travail, en l'absence de politiques industrielles, ne permettent pas de créer de nouveaux emplois, même dans les régions marquées par des économies relativement dynamiques ; pour les industries particulièrement exposées aux restructurations, la formation continue est cruciale, de même que l'amélioration de l'autonomie des travailleurs, notamment s'agissant des transitions entre les emplois ; compte tenu du report de l'âge de la retraite, la transition vers la retraite devrait être repensée, sauf à augmenter le nombre de travailleurs âgés au chômage et exposés au risque d'exclusion sociale.

Malgré les disparités entre les deux régions étudiées, la Vénétie et l'Emilie-Romagne, l'absence de caractérisation de genre des politiques du travail adoptées montre les limites de l'action publique. Cependant, il serait intéressant de poursuivre la recherche en termes comparatifs et d'observer les résultats de parcours si différenciés. 


\section{Bibliographie}

Ambrosini M., Coletto D. \& Guglielmi S. (2014), Perdere e ritrovare il lavoro. L'esperienza della disoccupazione al tempo della crisi, Bologna, Il Mulino.

Angeloff T. (1999), " Des miettes d'emploi : temps partiel et pauvreté », Travail, genre et sociétés, 1 , pp. 43-70.

Arber S., Ginn J. (1991), Gender and Later Life: A Sociological Analysis of Resources and Constraints, London, Sage.

Bagnasco A. \& Oberti M. (1998), “Le trompe-l'œil' of Regions”, in Lequesne C. \& Le Gales, (1998), Regions in Europe: The Paradox of Power, London, Routledge, pp. 114-125.

Bagnasco A. (1977), Tre Italie. La problematica territoriale dello sviluppo italiano, Bologna, Il Mulino.

Behaghel L., Caroli E. \& Roger M. (2014), "Age-biased technical and organizational change, training and employment prospects of older workers", Economica, 81(322), pp. 368-389.

Bisignano M. (2019), « Les politiques italiennes de l'emploi : une gestion des transitions professionnelles assurée par le chômage partiel ", Formation Emploi n 147, 3/2019.

Corsi M., Samek Lodovici M., Botti F. \& D’Ippoliti C. (2010), Active Ageing and Gender Equality Policies. Report for the European Commission, DG Employment, Social Affairs, and Equal Opportunities, disponible en ligne : http://www.sinisazrinscak.com/wpcontent/uploads/2012/12/EU-Active-Ageing-+-Gender-Report-2010.pdf [consulté le 18.09.2018].

Drury E. (1994), “Age Discrimination against Older Workers in the European Union”, The Geneva Papers on Risk and Insurance, 19, pp. 496-502.

Duncan C., Loretto W. (2004), "Never the Right Age? Gender and Age-based Discrimination in Employment", Gender, Work \& Organization, 1, pp. 95-115.

Fuchs Epstein C., Seron C., Oglensky B. \& Sauté R. (1981), The Part-Time Paradox: Time Norms, Professional Life, Family, and Gender, London, Routledge.

Giorgi F. (2018), "La recente evoluzione dell'indennità di disoccupazione in Italia ", Questioni di economia e finanza, (459).

Gist Y. J. \& Velkoff V. A. (1997), Gender and aging. Demographic dimensions, US Department of Commerce, Economics and Statistics Administration, Washington, DC 97-3, disponible en ligne : https://www.census.gov/library/publications/1997/ demo/ib97-3.html [Consulté le 18.09.2018]. 
ISFOL (2013), Caratteristiche strutturali ed evoluzione dell'offerta di formazione aziendale nel periodo 2005-2010. Dati INDACO-CVTS, disponible en ligne : http://isfoloa.isfol. it/handle/123456789/594 [consulté le 15.04.2019].

ISFOL (2015), XVI Rapporto sulla Formazione continua - Annualità 2014-2015, Ministero del lavoro e delle politiche sociali, Roma, disponible en ligne : http://bw5. cineca.it/bw5ne2/opac.aspx?WEB=INAP\&IDS=20746 [consulté le 15.09.2018].

Lee C. C., Czaja S. J. \& Sharit J. (2008), “Training older workers for technology-based employment", Educational Gerontology, 35(1), pp. 15-31.

Loretto W. \& al. (2007), The Future for Older Workers: New Perspectives, Bristol: Policy Press.

Maruani M. (1996), "L'emploi féminin à l'ombre du chômage ", Actes de la Recherche en Sciences Sociales, $\mathrm{n}^{\circ} 115$, pp. 48-57.

Moore S. (2009), “'No Matter What I Did I Would Still End Up in the Same Position'. Age as a Factor Defining Older Women's Experience of Labour Market Participation”, Work, Employment and Society, 23 (4), pp. 655-671.

Pleck J.H. (1977), “The Work-family Role System”, Social Problems, 24(4), pp. 417-427.

Reyneri E. \& Fullin G. (2013), "Gli immigrati in un mercato del lavoro in crisi”, Mondi migranti, 1, pp. 21-34.

Streeck W. (1989), "Skills and the Limits of Neo-Liberalism: The Enterprise of the Future as a Place of Learning", Work, Employment and Society, 3(1), pp. 89-104.

Trigilia C. (1986), Grandi partiti e piccole imprese: comunisti e democristiani nelle regioni a economia diffusa, Bologna, Il Mulino.

Urry J. (2014), Offshoring, Cambridge, Polity Press.

West K., Hussein A. \& Burke C. (2015), "Age Discrimination and Age Diversity Management within Vulnerable Groups”, in Jubany O. \& Perocco F. (édité par), Vulnerable Workers in Times of Social Transformations. Discrimination and Participation of Young and Older Workers, and Social Dialogue Stance, Venezia, Edizioni Ca' Foscari.

Williams van Rooij S. (2012), "Training older workers: Lessons learned, unlearned, and relearned from the field of instructional design”, Human Resource Management, 51, 2, pp. 281-298. 